\title{
The Effect of (E)-1-(4'-aminophenyl)-3-phenylprop-2-en-1-one on MicroRNA-18a, Dicer1, and MMP-9 Expressions against DMBA-Induced Breast Cancer
}

\author{
Ida Ayu Ika Wahyuniari ${ }^{1 *}$, I Gusti Kamasan Nyoman Arijana ${ }^{1}$, Ni Putu \\ Sriwidyani ${ }^{2}$, Hery Suwito ${ }^{3}$, Sitarina Widyarini ${ }^{4}$, Muhammad Ghufron ${ }^{5}$, Mustofa \\ Mustofa $^{6}$, Sofia Mubarika Haryana
}

\begin{abstract}
Background: Most of breast cancer patients are estrogen receptor alpha-positive and have high resistance and side effect of chemotherapeutic drug. Therefore, discovering an effective anticancer agent is needed. This research explored the effect of (E)-1-(4'-aminophenyl)-3-phenylprop-2-en-1-one (APE) on miR-18a, Dicer1, and MMP-9 expressions. Methods: Twenty four female Sprague-Dawley rats were invetigated in this study. The rats were divided into 6 groups of 4. G1 was considered as normal rat. G2, G3, T1, T2, and T3 were given DMBA $20 \mathrm{mg} / \mathrm{kgBW}$ twice a week for 5 weeks to induce mammary cancer. After being affiliated with cancer, G2 was given vehicle and G3 was treated with tamoxifen. T1, T2, and T3 were treated with APE intraperitoneally everyday for 21 days at doses of 5, 15, and 45 $\mathrm{mg} / \mathrm{kgBW} /$ day, respectively. Blood plasma was collected to measure $m i R-18 a$ expression using qRT-PCR. Mammary tissues were also collected to determine Dicerl and MMP-9 expressions by using immunohistochemistry. Results: The results showed significant down-regulation of miR-18a relative expression and up-regulation of Dicer 1 expression in $\mathrm{G} 3$ and $\mathrm{T} 1$ compared to $\mathrm{G} 2(\mathrm{P}<0.05)$. MMP-9 expression has significant decrease in $\mathrm{T} 1$ compared to $\mathrm{G} 2(\mathrm{P}<0.05)$. Conclusion: APE can decrease $m i R-18 a$ and $M M P-9$ expressions and increase Dicerl expression in rat mammary cancer. Therefore, this compound could be a candidate of novel anticancer.
\end{abstract}

Keywords: Chalcone- miR-18a-Dicer-MMP-9- mammary cancer

Asian Pac J Cancer Prev, 21 (5), 1213-1219

\section{Introduction}

Millions of women are diagnosed with breast cancer every year around the world. It is the most frequent malignancy in women (Akram et al., 2017; Zehni et al., 2019). Most of all breast cancer patients (70\%) are estrogen receptor alpha $(\mathrm{ER} \alpha)$-positive (Hayes and Lewis-Wambi, 2015; Zehni et al., 2019). Chemotherapeutic drugs, such as tamoxifen, have many undesirable side effects and their efficacy against carcinoma is still unsatisfactory. Nearly, $40-50 \%$ of patients with estrogen receptor-positive breast cancer develop resistance towards tamoxifen. In addition, the five-year survival rate after tamoxifen-resistance is less than $20 \%$. Tamoxifen-resistance is often presented in the form of tumor recurrence and/or distant metastasis in most cases (Tanic et al., 2012; Zhao and Ramaswamy,
2014; Rondon-Lagos et al., 2016; Sotgia et al., 2017). The recurrence rate of ER $\alpha$-positive breast cancer is $90 \%$ in Luminal B and 30\% in Luminal A (Sas et al., 2012). Therefore, much more attention on the discovery of effective anticancer agents is needed.

The medicinal chemists of natural product have been explored for drug discovery of potential anti-cancer agents, such as flavonoids given their antioxidant and cytotoxic properties (Pande et al., 2017). Flavonoid regulates $m i R N A$ expressions through epigenetic modification, transcription factors modulation, and miRNA maturation process (Srivastava et al., 2015). Chalcones belong to flavonoid family that has been shown to exert many properties for human diseases, including anticancer (Jin et al., 2013).

A series of new chalcone derivatives had been successfully synthesized to discover new anticancer, such as

${ }^{1}$ Department of Histology, Faculty of Medicine, Udayana University, Bali, Indonesia. 2Department of Anatomical Pathology, Faculty of Medicine, Udayana University, Bali, Indonesia. ${ }^{3}$ Department of Chemistry, Faculty of Science and Technology, Universitas Airlangga, Surabaya, Indonesia. ${ }^{4}$ Department of Pathology, Faculty of Veterinary Medicine, Universitas Gadjah Mada, Yogyakarta, Indonesia. ${ }^{5}$ Department of Histology and Cell Biology, Universitas Gadjah Mada, Yogyakarta, Indonesia. ${ }^{6}$ Department of Pharmacology and Therapy, Faculty of Medicine, Universitas Gadjah Mada, Yogyakarta, Indonesia. *For Correspondence: ikawahyuniari@unud.ac.id 
(e)-1-(4'-aminophenyl)-3-phenylprop-2-en-1-one (APE) (Suwito et al., 2015). Previously, the potential cytotoxicity of this compound against breast cancer cell line had been proven reducing tumor growth and down-regulating miR-21 in rat mammary cancer (Wahyuniari et al., 2017). However, lack of study regarding mechanism of action of this compound in metastasis effect.

Loss of Dicer expression is associated with the progression and metastasis of breast cancer (Khoshnaw et al., 2012). Dicer expression is significantly lower in triple-negative breast cancer (TNBC) versus estrogen receptor-positive $(\mathrm{ER}+)$ clinical specimen of primary tumor. Overall, TNBC has poor prognosis in comparison with ER+ breast cancer (Spoelstra et al., 2016). Dicer is a Ribonuclease III enzyme playing a crucial role in microRNAs (miRNA) maturation from pre-miRNAs molecules (Price and Chen, 2014). As we know, miRNA is an important epigenetic mechanism which acts as a negative gene regulator by binding to mRNA. Epigenetic is potential target in cancer treatment and prevention due to its modifiable nature (Basse and Arock, 2015). Furthermore, the activity of more than $60 \%$ of all protein-coding genes is predicted to be controlled by miRNAs in mammals (Catalanotto et al., 2016). Interestingly, Dicer1 is also regulated by miRNA. Hence, over-expression of miRNAs targeting Dicerl leads to global down-regulation of miRNA expression (Luo et al., 2013).

One of the miRNAs that regulates Dicer is miR-18a through its affinity with the 3' untranslated region (Luo et al., 2013; Chen et al., 2014). MiR-18a also regulates $\mathrm{ER} \alpha$ (Howard and Yang, 2018); however, Dicer is more significant than ER as a prognostic factor (Khoshnaw et al., 2012). In breast cancer, the expression of miR-18a increases (Shidfar et al., 2016), but the expression of Dicerl decreases (Yan et al., 2012). A previous study showed that increased $m i R-18 a$ expression targeting Dicer-1 in nasopharyngeal cancer led to $78 \%$ decreased miRNA expression, including miR-143 (Luo et al., 2013). Decrease in $m i R-143$ expression leads to increase of matrix metalloproteinase-9 (MMP-9) expression due to MMP-9 as target molecule of miR-143 (Abba et al., 2014). MMP-9 degrades protein in extracellular matrix and it is associated with tumor invasion, metastasis, and poor prognosis in breast cancer (Merdad et al., 2014; Yousef et al., 2014). In addition, the increase of $M M P-9$ expression can be also due to insufficiency of PTEN (Chiang et al., 2016), which is also a binding target for miR-18a (Zhang et al., 2016).

The current study aimed to investigate APE effect on miR-18a, Dicer1, and MMP-9 as molecular targets. Given that animal model may generate ER $\alpha$-positive breast cancer on Sprague Dawley rat (Abba et al., 2016; Alvarado et al., 2017), we first determined the molecular mechanism by which APE inhibited invasiveness against 7,12-dimethylbenz(a)anthracene (DMBA)-induced breast cancer.

\section{Materials and Methods}

\section{Tested compound and animals}

We tested (e)-1-(4'-aminophenyl)-3-phenylprop-2- en-1-one (APE) in this experimental research. It was synthesized by (Suwito et al., 2015) at Department of Chemistry, Faculty of Science and Technology, Universitas Airlangga, Indonesia. This in vivo research was a randomized post-test only control group design using twenty four female Sprague-Dawley rats with the aged of 3-4 weeks were provided by Laboratorium Penelitian dan Pengujian Terpadu, Universitas Gadjah Mada (UGM). This study was approved by Medical and Health Research Ethics Committee Faculty of Medicine, UGM. The rates were caged individually in animal house of the Department of Pharmacology and Therapy, Faculty of Medicine, UGM. They were maintained on a $12 \mathrm{~h}$ light-dark cycle at $24^{\circ} \mathrm{C}$. Rats were fed a standard diet. They were given ad libitum access to water.

The rats were randomly divided into 6 groups of 4 . Group 1 (G1) was treated with corn oil as control group. Other groups (T1, T2, T3, G2, G3) were given chemical carcinogen dimethylbenz(a)antracene (DMBA) 20 $\mathrm{mg} / \mathrm{kgBW}$ (Sigma-Aldrich, St Louis) dissolved in corn oil twice a week for five weeks to induce mammary cancer (Meiyanto et al., 2007). Tested compound was dissolved with vehicle (saline: tween 80 : DMSO $=8$ : 1: 1). Upon the appearance of mammary cancer, G2 (mammary cancer) was given vehicle and G3 (mammary cancer + tamoxifen) was treated with tamoxifen citrate (tamofen 10, Kalbe Farma, Indonesia) $6.6 \mathrm{mg} / \mathrm{kgBW}$. All $\mathrm{T}$ (mammary cancer $+\mathrm{APE}$ ) groups were treated with APE dissolved in vehicle intraperitoneally every day for 21 days at the doses of 5,15 , and $45 \mathrm{mg} / \mathrm{kgBW}$, respectively (Wahyuniari et al., 2017).

\section{Analysis of qRT-PCR}

Qiagen miRNeasy plasma kit (Cat\#217184) and miRNeasy plasma spike-in control (Cat\#219610) were used to extract microRNA in accordance with the manufacturer's protocol. Synthesis of cDNA was based on the protocol of Qiagen miscript II RT Kit (Cat\#218160). The level of miRNAs was quantified by using MyGo Mini Real-time PCR (IT-IS Life Science, UK) and Qiagen miScript SYBR Green PCR Kit (Cat\#218073). The primers used were rno-miR-18a specific primer, 5'-TAAGGTGCATCTAGTGCAGATAG-3', and miScript universal primer (IDT, Singapore). The miR-18a levels of plasma used C. elegans miR-39 as internal control. They were normalized with the comparative $\mathrm{Ct}$ method relative to this exogenous miRNA. The fold change in expression of the target gene relative to the internal control gene was calculated using $2^{-\Delta \Delta \mathrm{CT}}$ method (Wang et al., 2015b; Vigneron et al., 2016).

\section{Immunohistochemistry}

Twenty formalin fixed and paraffin-embedded blocks of rat mammary cancer were cut from G2, G3, T1, T2, and T3 (6 $\mu \mathrm{m}$ thick) and mounted on polylysine-coated slides. After deparaffinization in xylene, slides were rehydrated through graded series of alcohol and washed in phosphate buffer saline (PBS). The slides were steam for antigen retrieval and incubated in a protein block. Slides were incubated with polyclonal anti Dicer1 protein antibody (rabbit anti-rat Dicer1, Bioss, USA, Cat\#bs-6697R) with 
dilution 1: 100 and anti MMP-9 protein antibody (rabbit anti-rat MMP-9, Bioss, USA, Cat\#bs-0397R) with dilution 1: 200 based on manufacturer's instructions. Each IHC run included appropriate positive and negative controls. Immunohistochemical expression of Dicer 1 and MMP-9 was assessed using light microscope in five visual fields selected as previously mentioned (Wehrhan et al., 2012) with 400x magnification. The number of cytoplasmic staining according to its intensity of mammary cancer cells were used image raster 3 software. Intensity of the cytoplasmic staining and its distribution were assessed using the semi-quantitative scoring system (H-SCORE) The formula was as follows: H-SCORE $=\Sigma \mathrm{Pi}(\mathrm{i}+$ 1)/100, where Pi was the percentage for each intensity of mammary cancer cell's cytoplasmic staining (0-100\%) and $i$ was the staining intensity with a value of $0-3$ that referred to lack of, weak, moderate, and strong staining, respectively (Germeyer et al., 2014).

\section{Statistical analysis}

To analyze the relative expression of $m i R-18$ a, Dicerl, and MMP-9 expression, SPSS (version 17.0) was used and one way ANOVA was run. $\mathrm{P}<0.05$ was set for statistical
The Effect of (E)-1-(4'-aminophenyl)-3-phenylprop-2-en-1-one significance.

\section{Results}

\section{miR-18a expression}

The qRT-PCR examination revealed that $m i R-18 a$ had lower expression in all $\mathrm{T}$ groups compared to $\mathrm{G} 2$ $(\mathrm{G} 2=16.45 \pm 4.89), \mathrm{G} 3=5.65 \pm 2.29), \mathrm{T} 1=3.67 \pm 0.95)$, $\mathrm{T} 2=9.36 \pm 5.09$ ), and T3=10.80 \pm 5.89 ) (Figure 1A). Statistical analysis showed significant lower result were only in groups $\mathrm{G} 3(\mathrm{p}=0.011)$ and $\mathrm{T} 1(\mathrm{p}=0.002)$ compared to G2. However, T2 and T3 was not significant different ( $>0.05$ ) compared to G2.

\section{Dicer1 and MMP-9 expression}

Figure 2 shows different cytoplasmic staining intensities of Dicer 1 expression in 400x magnification. We detected loss of Dicer1 in untreated mammary cancer (G2). H-SCORE of Dicer 1 expression was higher in treatment groups compared to untreated groups ( $\mathrm{G} 2=0.63 \pm 0.44$, $\mathrm{G} 3=1.88 \pm 0.18$ vs. $\mathrm{T} 1=1.80 \pm 0.32, \mathrm{~T} 2=1.37 \pm 0.19$, and $\mathrm{T} 3=1.21 \pm 0.59)$. H-SCORE of Dicerl expression was significantly different in $\mathrm{G} 3$ and $\mathrm{T} 1(\mathrm{P}=0.002)$ (Figure 1B).

A

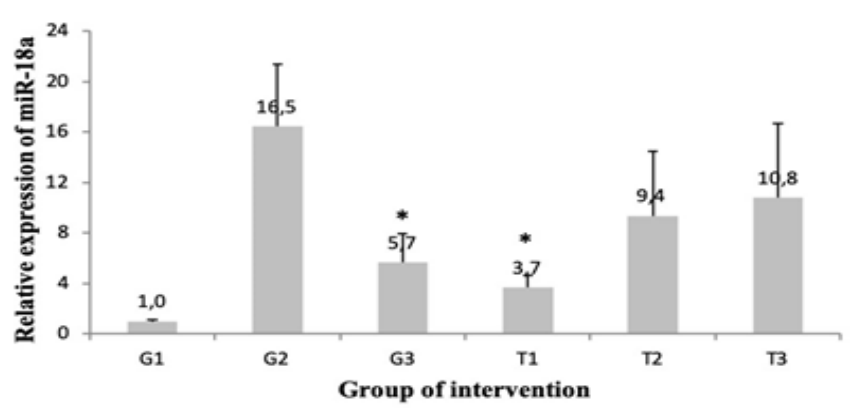

B
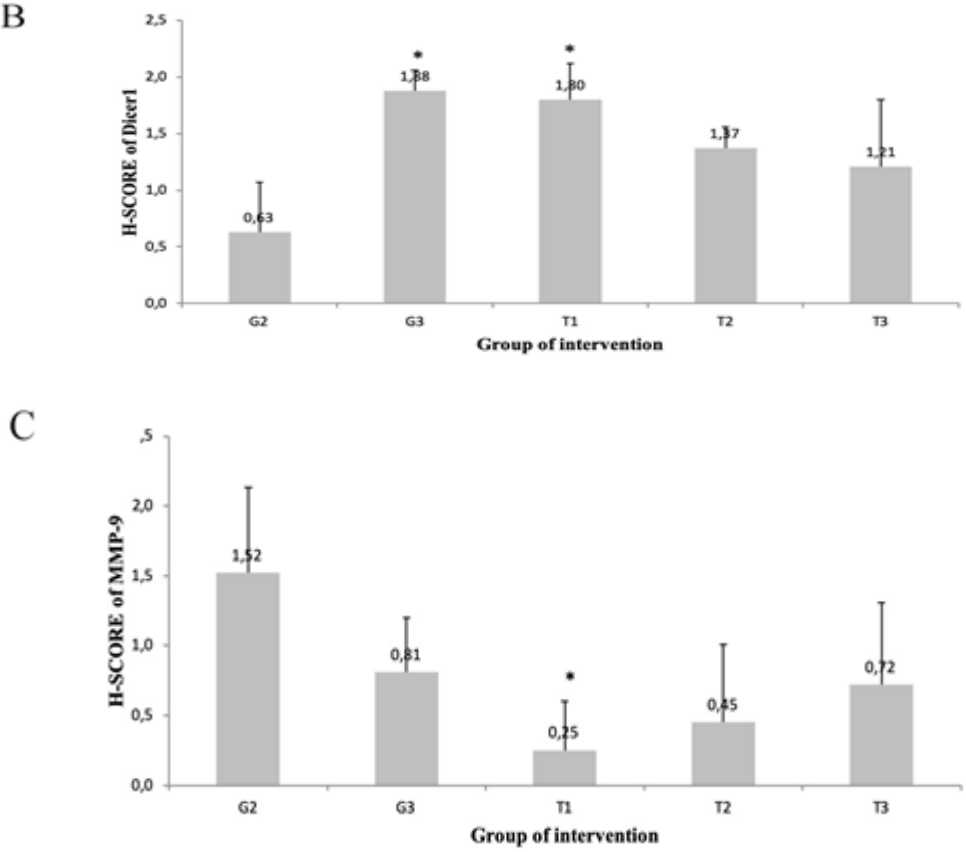

Figure 1. Effect of APE on miR-18a (A), Dicer1 (B), and MMP-9 (C) expression of rat mammary cancer. G1: normal rat, G2: mammary cancer rat, G3: mammary cancer + tamoxifen, T1: mammary cancer + APE 5 mg, T2: mammary cancer + APE $15 \mathrm{mg}$, T3: mammary cancer + APE $45 \mathrm{mg}$. *Significantly different from G2 $(\mathrm{P}<0.05)$. 

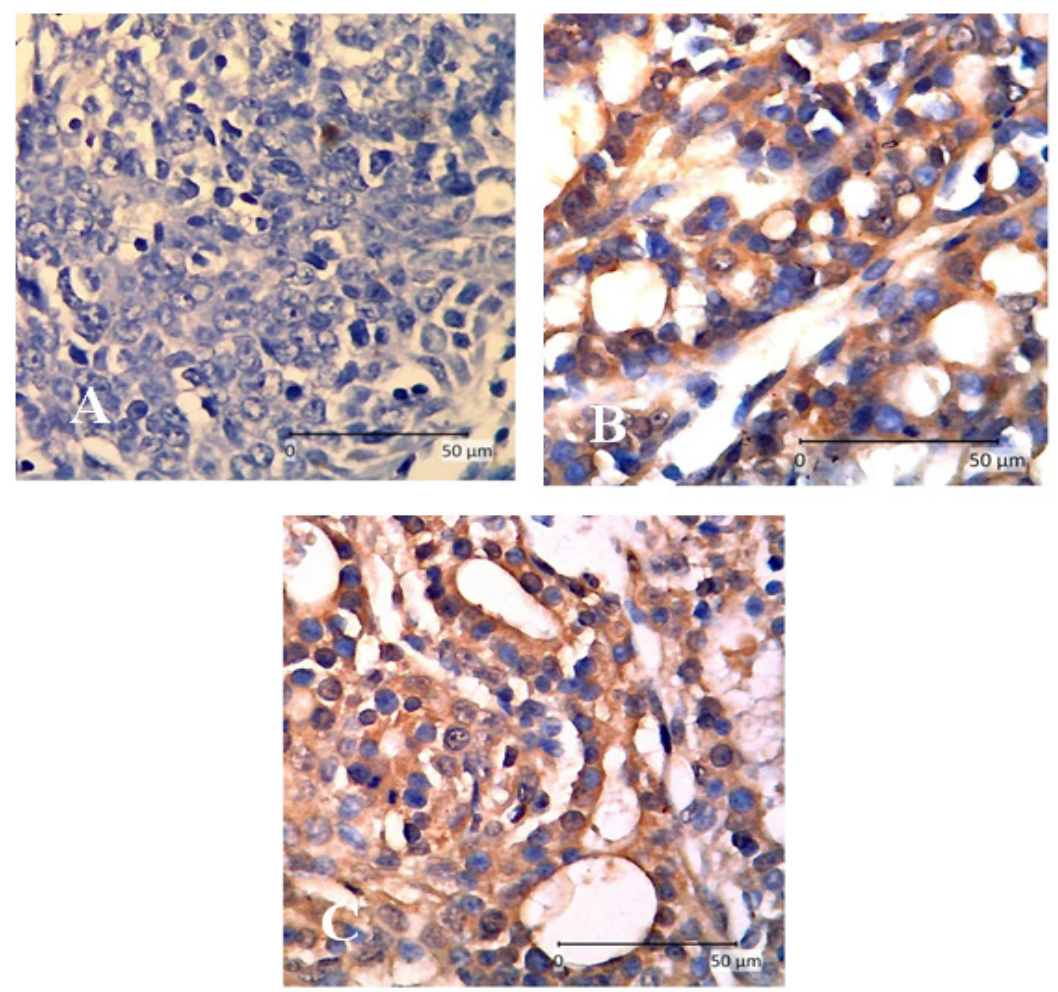

Figure 2. Dicer1 Expression in Rat Mammary Cancer with Immunohistochemistry (400x magnifications). A, mammary cancer rat (G2); B, mammary cancer + tamoxifen (G3); C, mammary cancer + APE (T1). Cytoplasmic staining was increased in treatment groups (B and $\mathrm{C})$.

According to Figure 1C, MMP-9 expression decreased in all treatment groups compared to untreated ones $(\mathrm{G} 2=1.52 \pm 0.61, \mathrm{G} 3=0.81 \pm 0.39$ vs. $\mathrm{T} 1=0.25 \pm$ $0.35, \mathrm{~T} 2=0.45 \pm 0.56$, and $\mathrm{T} 3=0.72 \pm 0.59)$. The results was statistically significant only in Group T1 compared to $\mathrm{G} 2$ ( $\mathrm{P}=0.030)$, while other groups were not significant $(\mathrm{P}>0.05)$. The expression of MMP-9 based on IHC in $\mathrm{G} 2$, G3, and T1 is shown in Figure 3, revealing prominent cytoplasmic staining of MMP-9 expression in group DMBA and vehicle.
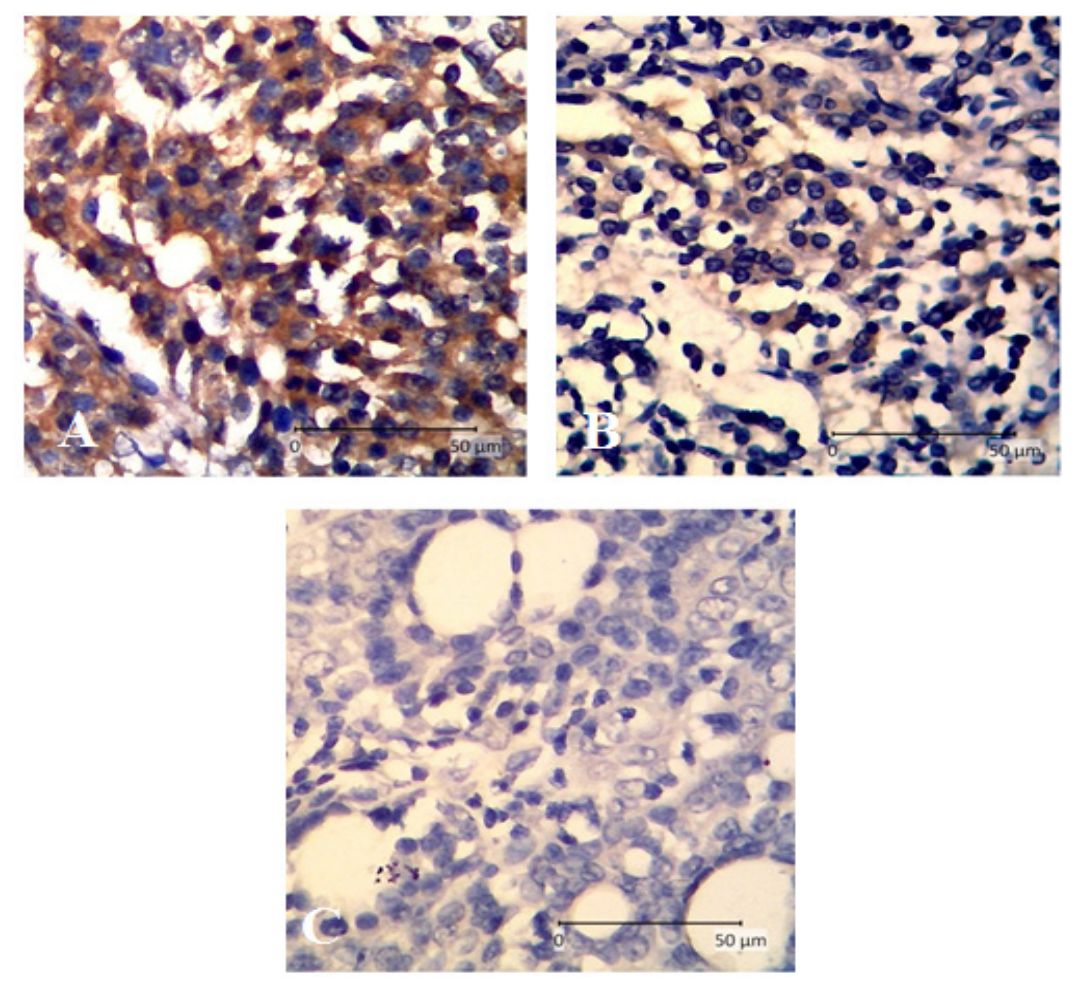

Figure 3. Immunohistochemistry of MMP-9 Expression in Rat Mammary Cancer (400x magnifications). A, mammary cancer (G2); B, mammary cancer + tamoxifen (G3); C, mammary cancer + APE (T1). Cytoplasmic staining was decreased in treatment groups (B and $\mathrm{C})$. 


\section{Discussion}

This study showed that APE at dose of $5 \mathrm{mg} / \mathrm{kgBW}$ could decrease $m i R-18 a$ expression in this in vivo study.

This compound belongs to chalcone derivatives as part of flavonoids family (Solomon and Lee, 2012). One study showed that flavonoids affected epigenetic mechanism including microRNA (Busch et al., 2015; Srivastava et al., 2015). Flavonoid regulates miRNA expression through modulation of transcription factors, epigenetic modification, and maturation of miRNA (Srivastava et al., 2015). This compound was also reported to down-regulate miR-21 (Wahyuniari et al., 2017). It was revealed that chrysin also down-regulated $m i R-221$, $m i R-21$, and $m i R-18 a$ expressions in gastric cancer (Mohammadian et al., 2016)

The expression of $m i R-18 a$ in this study was significantly higher in untreated rat mammary cancer (G2). Some studies showed similar results for breast cancer (Kodahl et al., 2014; Shidfar et al., 2016) and other cancers, such as nasopharyngeal cancer (Luo et al., 2013), gastric cancer (Tsujiura et al., 2015), esophageal cancer (Hirajima et al., 2013), pancreatic cancer (Morimura et al., 2011), colorectal cancer (Zhang et al., 2013; Yau et al., 2014), and hepatic cancer (Li et al., 2012). MicroRNA-18a is highly stable in blood; therefore, it is suitable as biomarker for non-invasive monitoring of tumor dynamics (Komatsu et al., 2014; Jin et al., 2015). MicroRNA can be a specific target for new anticancer agents due to its regulation on multiple target of mRNAs (Guo et al., 2013).

Dicer1 mRNA is regulated by miR-18a through its affinity with the 3' untranslated region of Dicer1 (Luo et al., 2013; Chen et al., 2014). In breast cancer, the expression of miR-18a increases (Shidfar et al., 2016) but the expression of Dicer1 decreases (Yan et al., 2012). In this study, it was found that APE at the dose of $5 \mathrm{mg} /$ kgBW significantly up-regulated Dicer 1 expression in T1. So, that is appropriate result that the decrease of $m i R-18 a$ expression is followed by the up regulation of Dicer1 in Group T1 due to APE administration. According to a previous study, Dicer expression was positively correlated with disease free interval in 5 years. It meant that those patients who had high Dicer expression were less likely to have recurrence in 5 years compared to low Dicer expression patients. Dicer was thought to be more significant as prognostic factor than estrogen receptor in breast cancer patients (Khoshnaw et al., 2012).

In this study, we found that Dicerl had the lowest expression in rat mammary cancer without treatment (G2). Another study showed a gradual decrease of Dicer during progression of breast cancer, which was the strongest Dicer expression found in normal breast epithelial cells and the weakest in metastatic cells (Khoshnaw et al., 2012). Given that Dicer plays a crucial role in final maturation of miRNA (Price and Chen, 2014), Dicer dysregulation can lead to global disruption of miRNA expressions. Interestingly, Dicer1 is also regulated by miRNA. One study showed that up-regulation of miR-18a suppressed Dicer expression, causing global down-regulation of miRNA expression (78\%), such as miR-143 (Luo et al., 2013). One of miR-143 target molecule is MMP-9 that
The Effect of (E)-1-(4'-aminophenyl)-3-phenylprop-2-en-1-one

is associated with tumor invasion, metastasis, and poor prognosis of breast cancer via protein degradation in extracellular matrix (Merdad et al., 2014; Yousef et al., 2014).

In this study, anti-invasive control by APE was confirmed by the measurement of $M M P-9$ expression. Down-regulation of MMP-9 was detected in groups treated with APE in 3 variation of dosage and tamoxifen, but significant only in group APE with dose $5 \mathrm{mg} / \mathrm{kgBW}$. In addition, MMP-9 down-regulation may be also due to PTEN up-regulation which is also a binding target for miR-18a. We found APE capability to inhibit cancer progression. Other chalcone derivatives showed the same manner in metastasis inhibition. Synthetic chalcone, E-2-(40-methoxybenzylidene)-1-benzosuberone decreased secretion of MMP-9 (Pilatova et al., 2010). However, $M M P-2$ expression was also decreased by novel anthraquinone based chalcone analogue (Kolundzija et al., 2014) and 2, 2-dimethylbenzopyran (Wang et al., 2015a).

The best response of APE was found in lower dosage. High dose of flavonoids may be toxic given their prooxidant activity and pro-inflammatory effects (Galati and O'Brien, 2004; Bouayed and Bohn, 2010; Corcoran et al., 2012). Further studies are suggested to find the optimal dosage of APE considering better efficacy of its lower dosage.

In conclusion, this study suggested that (E)-1-(4'aminophenyl)-3-phenylprop-2-en-1-one (APE) may decrease miR-18a and increase Dicer1. This compound could inhibit invasiveness of breast cancer by decreasing MMP-9 expression. These results are substantial for the future development of this compound to control metastasis that often occurs in resistance and recurrence of breast cancer .

\section{Acknowledgements}

Grant from Research and Development Unit, Faculty of Medicine, Udayana University, Bali-Indonesia funded part of this research.

\section{References}

Abba M, Patil N, Allgayer H (2014). MicroRNAs in the regulation of MMPs and metastasis. Cancers (Basel), 6 , 625-45.

Abba MC, Zhong Y, Lee J, et al (2016). DMBA induced mouse mammary tumors display high incidence of activating Pik3caH1047 and loss of function PTEN mutations. Oncotarget, 7, 64289-99.

Akram M, Iqbal M, Daniyal M, et al (2017). Awareness and current knowledge of breast cancer. Biol Res, $\mathbf{5 0 .}$

Alvarado A, Lopes AC, Faustino-Rocha AI, et al (2017). Prognostic factors in MNU and DMBA-induced mammary tumors in female rats. Pathol Res Pract, 213, 441-6.

Basse C, Arock M (2015). The increasing roles of epigenetics in breast cancer: Implications for pathogenicity, biomarkers, prevention and treatment. Int J Cancer, 137, 2785-94.

Bouayed J, Bohn T (2010). Exogenous antioxidants--Doubleedged swords in cellular redox state: Health beneficial effects at physiologic doses versus deleterious effects at high doses. Oxid Med Cell Longev, 3, 228-37.

Busch C, Burkard M, Leischner C, et al (2015). Epigenetic Asian Pacific Journal of Cancer Prevention, Vol $21 \quad 1217$ 
activities of flavonoids in the prevention and treatment of cancer. Clin Epigenetics, 7, 64.

Catalanotto C, Cogoni C, Zardo G (2016). MicroRNA in control of gene expression: An overview of nuclear functions. Int $J$ Mol Sci, 17.

Chen X, Wang J, Cheng L, et al (2014). MiR-18a downregulates DICER 1 and promotes proliferation and metastasis of nasopharyngeal carcinoma. Int J Clin Exp Med, 7, 847-55.

Chiang K, Hsu S, Lin S, et al (2016). PTEN insufficiency increases breast cancer cell metastasis in vitro and in vivo in a xenograft Zebrafish Model. Anticancer Res, 36, 3997-4006.

Corcoran MP, McKay DL, Blumberg JB (2012). Flavonoid basics: chemistry, sources, mechanisms of action, and safety. J Nutr Gerontol Geriatr, 31, 176-89.

Galati G, O'Brien PJ (2004). Potential toxicity of flavonoids and other dietary phenolics: significance for their chemopreventive and anticancer properties. Free Radic Biol Med, 37, 287-303.

Germeyer A, Savaris RF, Jauckus J, et al (2014). Endometrial beta3 integrin profile reflects endometrial receptivity defects in women with unexplained recurrent pregnancy loss. Reprod Biol Endocrinol, 12, 53.

Guo L, Zhao Y, Yang S, et al (2013). Genome-wide screen for aberrantly expressed miRNAs reveals miRNA profile signature in breast cancer. Mol Biol Rep, 40, 2175-86.

Hayes EL, Lewis-Wambi JS (2015). Mechanisms of endocrine resistance in breast cancer: an overview of the proposed roles of noncoding RNA. Breast Cancer Res, 17, 40.

Hirajima S, Komatsu S, Ichikawa D, et al (2013). Clinical impact of circulating miR-18a in plasma of patients with oesophageal squamous cell carcinoma. Br J Cancer, 108, 1822-9.

Howard EW, Yang X (2018). microRNA regulation in estrogen receptor-positive breast cancer and endocrine therapy. Biol Proced Online, 20, 17.

Jin C, Liang YJ, He H, et al (2013). Synthesis and antitumor activity of novel chalcone derivatives. Biomed Pharmacother, 67, 215-7.

Jin S, Tan S, Li H (2015). MicroRNA-18a as a promising biomarker for cancer detection: a meta-analysis. Int J Clin Exp Med, 8, 12286-96.

Khoshnaw SM, Rakha EA, Abdel-Fatah TM, et al (2012). Loss of dicer expression is associated with breast cancer progression and recurrence. Breast Cancer Res Treat, 135, 403-13.

Kodahl AR, MB L, H B, et al (2014). Novel circulating microRNA signature as a potential non-invasive multi-marker test in ER-positive early-stage breast cancer: a case control study. Mol Oncol, 8, 874-83.

Kolundzija B, Markovic V, Stanojkovic T, et al (2014). Novel anthraquinone based chalcone analogues containing an imine fragment: synthesis, cytotoxicity and anti-angiogenic activity. Bioorg Med Chem Lett, 24, 65-71.

Komatsu S, Ichikawa D, Takeshita H, et al (2014). Circulating miR-18a: a sensitive cancer screening biomarker in human cancer. In Vivo, 28293-8.

Li L, Guo Z, Wang J, et al (2012). Serum miR-18a: a potential marker for hepatitis B virus-related hepatocellular carcinoma screening. Dig Dis Sci, 57, 2910-6.

Luo Z, Dai Y, Zhang L, et al (2013). MiR-18a promotes malignant progression by impairing microRNA biogenesis in nasopharyngeal carcinoma. Carcinogenesis, 34, 415-25.

Meiyanto E, Tasminatun S, Susilowati S, et al (2007). Suppression of DMBA-induced carcinogenesis of breast cancer in post initition stage by ethanolic extract of Gynura procumbens (Lour), Merr leaves. Indonesian J Pharm, 18, 169-75.

Merdad A, Karim S, Schulten HJ, et al (2014). Expression of
Matrix Metalloproteinases (MMPs) in primary human breast cancer: MMP-9 as a potential biomarker for cancer invasion and metastasis. Anticancer Res, 34, 1355-66.

Mohammadian F, Pilehvar-Soltanahmadi Y, Mofarrah M, et al (2016). Down regulation of miR-18a, miR-21 and miR-221 genes in gastric cancer cell line by chrysin-loaded PLGAPEG nanoparticles. Artif Cells Nanomed Biotechnol, 44, 1972-8.

Morimura R, Komatsu S, Ichikawa D, et al (2011). Novel diagnostic value of circulating miR-18a in plasma of patients with pancreatic cancer. Br J Cancer, 105, 1733-40.

Pande AN, Biswas S, Reddy ND, et al (2017). In vitro and in vivo anticancer studies of 2'-hydroxy chalcone derivatives exhibit apoptosis in colon cancer cells by HDAC inhibition and cell cycle arrest. EXCLIJ, 16, 448-63.

Pilatova M, Varinska L, Perjesi P, et al (2010). In vitro antiproliferative and antiangiogenic effects of synthetic chalcone analogues. Toxicol In Vitro, 24, 1347-55.

Price C, Chen J (2014). MicroRNAs in cancer biology and therapy: Current status and perspective. Genes Dis, 1, 53-63.

Rondon-Lagos M, Villegas VE, Rangel N, et al (2016). Tamoxifen resistance: Emerging molecular targets. Int $J$ Mol Sci, 17.

Sas L, Lardon F, Vermeulen PB, et al (2012). The interaction between ER and NFкB in resistance to endocrine therapy. Breast Cancer Res, 14, 212-26.

Shidfar A, Costa FF, Scholtens D, et al (2016). Expression of miR-18a and miR-210 in normal breast tissue as candidate biomarkers of breast cancer risk. Cancer Prev Res, 10, 89-97.

Solomon VR, Lee H (2012). Anti-breast cancer activity of heteroaryl chalcone derivatives. Biomed Pharmacother, 66, 213-20.

Sotgia F, Fiorillo M, Lisanti MP (2017). Mitochondrial markers predict recurrence, metastasis and tamoxifen-resistance in breast cancer patients: Early detection of treatment failure with companion diagnostics. Oncotarget, 8, 68730-45.

Spoelstra NS, Cittelly DM, Christenson JL, et al (2016). Dicer expression in estrogen receptor-positive versus triple-negative breast cancer: an antibody comparison. Hum Pathol, 56, 40-51.

Srivastava SK, Arora S, Averett C, et al (2015). Modulation of microRNAs by phytochemicals in cancer: underlying mechanisms and translational significance. Biomed Res Int, 2015, 848710 .

Suwito H, Jumina, Mustofa, et al (2015). Anticancer and antimicrobial activity of methoxy amino chalcone derivatives. Der Pharma Chemica, 7, 89-94.

Tanic N, Milovanovic Z, Dzodic R, et al (2012). The impact of PTEN tumor suppressor gene on acquiring resistance to tamoxifen treatment in breast cancer patients. Cancer Biol Ther, 13, 1165-74.

Tsujiura M, Komatsu S, Ichikawa D, et al (2015). Circulating miR-18a in plasma contributes to cancer detection and monitoring in patients with gastric cancer. Gastric Cancer, 18, 271-9.

Vigneron N, Meryet-Figuiere M, Guttin A, et al (2016). Towards a new standardized method for circulating miRNAs profiling in clinical studies: Interest of the exogenous normalization to improve miRNA signature accuracy. Mol Oncol, 10, 981-92.

Wahyuniari IAI, Arijana IGKN, Sriwidyani NP, et al (2017). The anticancer activity of (e)-1-(4'-aminophenyl)-3-phenylprop2-en-1-one against DMBA-induced mammary cancer in Sprague Dawley rat through the regulation of microRNA-21 expression. Bali Med J, 6, 589.

Wang L, Chen G, Lu X, et al (2015a). Novel chalcone derivatives as hypoxia-inducible factor (HIF)-1 inhibitor: synthesis, anti-invasive and anti-angiogenic properties. Eur J Med 
Chem, 89, 88-97.

Wang P, Yang D, Zhang H, et al (2015b). Early detection of lung cancer in serum by a panel of MicroRNA biomarkers. Clin Lung Cancer, 16, 313-9 e1.

Wehrhan F, Amann K, Molenberg A, et al (2012). PEG matrix enables cell-mediated local BMP-2 gene delivery and increased bone formation in a porcine critical size defect model of craniofacial bone regeneration. Clin Oral Implants Res, 23, 805-13.

Yan M, Huang HY, Wang T, et al (2012). Dysregulated expression of dicer and drosha in breast cancer. Pathol Oncol Res, 18, 343-8.

Yau TO, Wu CW, Dong Y, et al (2014). microRNA-221 and microRNA-18a identification in stool as potential biomarkers for the non-invasive diagnosis of colorectal carcinoma. $\mathrm{Br}$ J Cancer, 111, 1765-71.

Yousef EM, Tahir MR, Pierre YS, et al (2014). MMP-9 expression varies according to molecular subtypes of breast cancer. BMC Cancer, 14, 609-20.

Zehni AZ, Jacob SN, Mumm JN, et al (2019). Hormone receptor expression in multicentric/multifocal versus unifocal breast cancer: Especially the VDR determines the outcome related to focality. Int J Mol Sci, 20, 1-17.

Zhang GJ, Zhou T, Liu ZL, et al (2013). Plasma miR-200c and miR-18a as potential biomarkers for the detection of colorectal carcinoma. Mol Clin Oncol, 1, 379-84.

Zhang W, Lei C, Fan J, et al (2016). miR-18a promotes cell proliferation of esophageal squamous cell carcinoma cells by increasing cylin D1 via regulating PTEN-PI3K-AKT-mTOR signaling axis. Biochem Biophys Res Commun, 477, 144-9.

Zhao M, Ramaswamy B (2014). Mechanisms and therapeutic advances in the management of endocrine-resistant breast cancer. World J Clin Oncol, 5, 248-62.

\section{c) (1) (8)}

This work is licensed under a Creative Commons AttributionNon Commercial 4.0 International License. 